\title{
Factores demográficos relacionados con el apego materno infantil
}

\author{
Demographic factors related to maternal and child attachment
}

Dora Isabel Giraldo-Montoya1* orcid.org/0000-0003-0324-3975

Hellen Lucia Castañeda-Palacio' ${ }^{1}$ orcid.org/0000-0001-8065-5514

Héctor Mauricio Mazo-Álvarez¹ orcid.org/0000-0002-5096-6618

1 Universidad Pontificia Bolivariana. Medellín, Colombia

Fecha de recepción: Octubre 31 - 2016

Fecha de revisión: Febrero 8 - 2017

Fecha de aceptación: Agosto 11 - 2017

Giraldo-Montoya DI, Castañeda-Palacio HL, Mazo-Álvarez HM. Factores demográficos relacionados con en el apego materno infantil. Univ. Salud. 2017;19(2):197-206. DOI: http://dx.doi.org/10.22267/rus.171902.82

\section{Resumen}

Introducción: El postparto es uno de los momentos determinantes para fortalecer el apego entre la diada madre e hijo, sin embargo existen factores demográficos que pueden limitar esta interacción y convertirse en una desventaja para el desarrollo adecuado del niño. Objetivo: Determinar los factores demográficos relacionados con el apego materno infantil en el postparto, durante el año 2014. Materiales y Métodos: Estudio analítico, transversal, muestra conformada por 117 madres con su recién nacido. Se utilizó el instrumento Mother-child relationship. Las variables para determinar el apego fueron: lactancia materna, interés, la respuesta, lenguaje y tacto; evaluadas en una escala numérica cuyo puntaje final permitió determinar si existía apego sano o en riesgo. Se realizó un modelo de regresión logística para explorar la asociación entre variables demográficas y el apego en riesgo. Resultados: La mediana de edad fue de 27 años, rango intercuartílico entre 22 y 31 años, 54,7\% vivían en unión libre y 34,2\% tenían bachillero completo. El apego sano se presentó en 80,3\% de las madres; sin embargo, el 60,7\% tuvieron baja motivación inicial hacia la lactancia lo cual podría generar un apego en riesgo. Se encontró asociación estadística entre apego en riesgo, estado civil y escolaridad, pero la única variable que explicó dicha asociación fue el estado civil soltero (RP=4,88 IC95\% p=0,48). Conclusión: De acuerdo al modelo multivariado, el apego en riesgo se asocia con el estado civil soltero.

Palabras clave: Periodo postparto; recién nacido; relaciones madre-hijo. (Fuente: DeCS, Bireme).

\begin{abstract}
Introduction: Postpartum is one of the decisive moments to strengthen the attachment between the mother and child relationship; however, there are demographic factors that can limit this interaction and become a disadvantage for the proper development of the child. Objective: Determine the demographic factors related to maternal child attachment in the postpartum during the year 2014. Materials and methods: An analytical transversal study was carried out with a sample of 117 mothers with their newborn. The instrument Mother-child relationship was used. The variables to determine attachment were: breastfeeding, interest, response, language and tact. They were evaluated on a numerical scale whose final score allowed to determine whether there was a healthy or at-risk attachment. A logistic regression model was implemented to explore the association between demographic variables and at-risk attachment. Results: The median age was 27 years with an interquartile range between 22 and $31.54 .7 \%$ lived in free union and $34.2 \%$ had fulfilled high school studies. Healthy attachment was presented in $80.3 \%$ of the mothers. Nevertheless, $60.7 \%$ had low initial motivation for breastfeeding which could lead to at-risk attachment. Statistical association between at-risk attachment, marital status and schooling was found, but the only variable that explained this association was the civil status: single (RP=4.88 IC95\% p=0.48). Conclusion: According to the multivariate model, at-risk attachment is associated with the civil status: single.
\end{abstract}

*Autor de correspondencia

Dora Isabel Giraldo Montoya

e-mail: dora.giraldo@upb.edu.co 
Keywords: Postpartum period, newborn, Mother-Child relationship. (Source: DeCS, Bireme).

\section{Introducción}

El postparto es uno de los momentos más relevantes para fortalecer el apego entre la diada madre e hijo. Este período es de gran importancia para brindar apoyo apropiado a la madre y su familia y para incentivar la interacción temprana, con el propósito de favorecer a futuro un desarrollo personal adecuado en el niño(1).

Los factores que influyen en el apego son variados, entre ellos: la personalidad, la salud física, la estructura corporal, la cultura, el apoyo social de la madre, la edad, el estado civil, las condiciones económicas y el nivel académico(2). Estos aspectos se relacionan con el ambiente que la madre le puede proporcionar al feto y al recién nacido y afectan su capacidad para relacionarse adecuadamente con su hijo. Las relaciones con otras personas importantes en su vida también afectarán la relación con el feto y el recién nacido². $\mathrm{Si}$ las experiencias tempranas del bebé con su madre, no son adecuadas, podría afectar el desarrollo mental del infante y del adulto; además repercute en sus relaciones interpersonales en la transición de la niñez a la adolescencia y posteriormente a la adultez ${ }^{(3)}$.

La teoría del apego desarrollada por Jhon Bowlby 4 , no solo brinda referentes conceptuales para comprender situaciones de riesgo en el desarrollo del apego, sino también, para puntualizar intervenciones de cuidado de enfermería efectivas y fortalecer el vínculo materno infantil en el postparto ${ }^{(2,4)}$. El estudio de Castillo $^{3}$, refiere que la calidad del apego madrehijo dependerá entonces, de lo que cada una de las partes involucradas aporte a la relación, así como de la influencia directa que cada una de ellas ejerce sobre la otra. Plantea cuatro fases de la conducta materna que son indicadores de una interacción adecuada entre madre e hijo, adaptadas desde Bowlby(3,4): "1) Contacto físico frecuente y sostenido entre el bebé y la madre, en especial durante los 6 primeros meses, y habilidad de la madre para apaciguar a un bebé ansioso sosteniéndolo en brazos; 2) Sensibilidad de la madre ante las señales del bebé y, en particular, su habilidad para sincronizar sus intervenciones de acuerdo con el ritmo de aquel; 3) Ambiente regulado de tal manera que el bebé puede percibir las consecuencias de sus propias acciones; 4) Deleite mutuo entre la madre e hijo cuando están juntos. Indicador que quizás sea tanto el resultado de las citadas anteriormente como condición en sí”.

Para identificar estas conductas y definir si corresponden a un apego sano o en riesgo, el estudio de Salariya y Cater validó la escala denominada Mother-child relationship-FIRST score $^{2}$, determinando las conductas indicadoras de una relación de riesgo entre madre e hijo, a través de la observación que hizo el personal de enfermería en el postparto inmediato. Evaluaron las siguientes conductas maternas: la alimentación (lactancia materna), el interés, la respuesta, el lenguaje y el tacto. Para aquellas madres que puntearon con apego en riesgo, estimaron la relación con las variables sociodemográficas como edad, estado civil, ocupación, escolaridad y la clase social y encontraron que la edad, el estado civil y la clase social, influyeron en la relación de apego entre madre e hijo. Otros estudios evaluaron la asociación entre las variables sociodemográficas y el apego, concluyendo que el nivel socioecómico, la escolaridad y la edad influyeron en las conductas de apego entre madre e hijo(5-7).

En este sentido, para identificar a las madres con apego sano o en riesgo, el presente estudio partió del concepto de Salariya y Cater quienes afirman que un apego sano se evidencia cuando la madre reconoció $y$ fue capaz de satisfacer la alimentación del bebé y necesitó una supervisión mínima o nula, se interesó en el bienestar del bebé y su desarrollo, celebró con el bebé cara a cara y habló con él, trató al bebé como si fuera capaz de comprender su conversación e interpretar las expresiones faciales, confió que sus respuestas le ofrecieron bienestar a su bebé e hizo contacto prolongado tocando, acariciando, besando o abrazando al bebé(2). 
Con el fin de identificar el apego materno infantil y los factores que influyen en él, se desarrolló el presente estudio en una Unidad de Salud de la ciudad de Medellín, la cual es reconocida en el medio, por su calidad y humanización en la atención del parto de bajo y de alto riesgo obstétrico. El flujo de maternas es alto, todas ellas con diferentes niveles educativos, estratos socioeconómicos, especialmente jóvenes y con diversas situaciones psicosociales, que pueden afectar las conductas de apego y la interacción con su recién nacido. El personal de enfermería que labora en el servicio de postparto, ha observado que algunas madres manifiestan poco interés por su hijo que acaba de nacer y muestran conductas que no favorecen al apego materno infantil. Así mismo, los estudiantes de enfermería de pregrado y postgrado, quienes cumplen sus objetivos de aprendizaje, han manifestado la necesidad de identificar aquellas diadas (madre e hijo) que por alguna circunstancia no realizan adecuadamente una interacción temprana con el recién nacido, ni tampoco se ven comprometidas con la lactancia materna, teniendo en cuenta que lactar es una de las mejores medidas para fomentar y fortalecer el apego. De la misma manera, mencionan su interés por saber más sobre el fortalecimiento del vínculo en las madres que acaban de tener a su hijo, a través de planes de cuidado puntuales para este grupo poblacional.

En este sentido, el grupo de investigadores del presente trabajo, conformado por un psicólogo dos enfermeras y la participación de dos estudiantes de posgrado, se planteó la pregunta ¿Cuáles son los factores que influyen en el apego materno infantil en el postparto?; teniendo en cuenta este cuestionamiento, se planteó como objetivo determinar los factores demográficos relacionados con el apego materno infantil en el postparto, durante el año 2014. Esto permitió comprender las situaciones de riesgo para el desarrollo adecuado del vínculo y también para puntualizar intervenciones, fortalecer guías de evidencia científica de cuidado efectivas y la relación materno - infantil.

\section{Materiales y métodos}

\section{Tipo de estudio}

Estudio analítico, transversal. La población estuvo constituida por 756 madres y recién nacidos cuyo parto fue atendido en una Unidad Materno Infantil de una clínica de tercer nivel de complejidad de Medellín, Colombia, durante el segundo semestre del año 2014.

\section{Tamaño de la muestra}

Se determinó el tamaño de muestra teniendo en cuenta una prevalencia de apego en riesgo del $30 \%$ según estudios previos (8-10), se trabajó con un nivel de confianza del 95\% y un nivel de precisión del 5\%, para un total de 117 madres con sus recién nacidos, quienes participaron de manera voluntaria en el estudio.

\section{Criterios de inclusión}

- Diada madre e hijo, hospitalizadas en postparto mediato (después de 24 horas del nacimiento).

- Madre con un postparto normal.

- Madre mayor de 18 años de edad.

- Madre con una gestación a término.

- Recién nacidos a término

\section{Criterios de exclusión}

- Madre con trastornos mentales o problemas de salud que requirieron de hospitalización.

- Recién nacido que presentó problemas de salud que requirieron de hospitalización.

\section{Instrumentos}

- El instrumento que se utilizó para medir las variables conductuales fue el denominado Mother-child relationship - FIRST score validado por Salariya EM y Cater JI en 1984(2).

- Se utilizó una encuesta para medir las variables demográficas y obstétricas, la cual fue diseñada por los investigadores considerando los objetivos del estudio. 


\section{Variables}

1. Conducta maternal definida de la siguiente manera:

a) Alimentación: Comprensión de la madre de la necesidad de alimentar al bebé a través de la lactancia materna.

b) Interés: Atención general en todos los niveles, de las necesidades del bebé. Respuesta: reacción de la madre y la forma de responder a las necesidades del bebé.

c) Lenguaje: Comunicación, verbal y no verbal (incluyendo el contacto visual).

d) El tacto: Tocar afectivamente durante el baño y / o cambio de pañal.

2. Demográficas y obstétricas: edad, estado civil, estrato socioeconómico, escolaridad, ocupación, edad gestacional, paridad, vía del parto.

El instrumento FIRST score(2) fue interpretado en una escala numérica donde:

- 0 = La madre no mostró las actitudes y comportamientos normales durante las actividades cotidianas del postparto.

- 1 = La madre mostró mínimamente las actitudes y comportamientos normales durante las actividades cotidianas del postparto.

- 2 = La madre mostró totalmente las actitudes y comportamientos normales durante las actividades cotidianas del postparto.

\section{Recolección de la información}

Se realizó un entrenamiento de los investigadores sobre:

- La utilización de la escala FIRST(2)

- Las conductas y comportamientos de las madres que suelen presentarse en el desarrollo del apego de acuerdo con la literatura y la práctica clínica.

Dos investigadores observaron simultáneamente las actitudes y comportamientos de la madre durante las actividades cotidianas del postparto: cambiar al bebé, vestirlo, alimentarlo, bañarlo, cargarlo, su comportamiento durante la vacunación y la revisión por el pediatra. El tiempo de observación fue en promedio 45 minutos para cada diada.

Para garantizar la reproducibilidad, los investigadores evaluaron al mismo tiempo las acciones de la madre y el bebé, luego, de manera conjunta, reflexionaron y analizaron las actitudes y comportamientos de la madre y posteriormente definieron el puntaje de la escala. De la misma manera, se tomaron fotografías que sirvieron para analizar y confrontar algunas actitudes y comportamientos y para validar los datos obtenidos en el instrumento.

Se realizó una prueba piloto a doce madres con sus bebés que permitió mejorar la evaluación de las conductas maternales y generar confianza y familiaridad con los parámetros empleados en el instrumento.

\section{Análisis estadístico}

A las variables cuantitativas se les estimó la media acompañada de su desviación estándar o la mediana con su rango intercuartílico (RIC) dependiendo de la distribución de los datos. A las variables cualitativas se les calculó frecuencias absolutas y relativas.

El total de las puntuaciones de la evaluación de las conductas (alimentación, lenguaje, tacto, interés y respuesta) se distribuyeron en grupos de 0-6 puntos para las madres con apego en riesgo y de 7 y más puntos para las madres con apego sano, según los criterios definidos por la escala Motherchild relationship - FIRST score ${ }^{2}$.

Se exploró la asociación entre la variable dependiente (apego) con las variables demográficas clínicas de interés, dicha asociación se estableció mediante Razones de Prevalencia (RP) con su respectivo intervalo de confianza del 95\%; y se aplicó la prueba de Chi cuadrado para determinar la significación estadística de la asociación, un valor $\mathrm{p}<0,05$, se consideró estadísticamente significativo. Posteriormente, se realizó una regresión logística a través del método enter para identificar las variables explicativas independientes para el apego, en la que ingresaron al modelo aquellas variables cuyo 
nivel de significancia en el análisis bivariado fue $<0,25$ de acuerdo con los criterios de HosmerLemeshow, o las que a pesar de no cumplir dicho criterio, tenían plausibilidad biológica para ingresar al modelo. Para la tabulación y análisis de la información se utilizó el software SPSS $^{\circledR}$, versión 24.

\section{Consideraciones éticas}

El estudio fue aprobado por el Comité de Ética de la Escuela de Ciencias de la Salud y por el Comité de Investigación de la Clínica. Según la Resolución № 008430 de 1993 de Colombia11, fue una investigación con riesgo mínimo. Todas las madres firmaron el consentimiento informado. Con el fin de salvaguardar la intimidad de las participantes del estudio, el registro fotográfico fue evaluado por ellas mismas y las fotografías que causaban incomodidad fueron eliminadas frente a ellas de manera inmediata.

\section{Resultados}

\section{Variables demográficas}

Las madres que participaron del estudio, tuvieron una mediana para la edad de 27 años, con un RIC entre 22 y 31 años. La distribución de las madres según el estrato socioeconómico mostró que el $51,3 \%$ de ellas pertenece al estrato uno, $46,2 \%$ al estrato tres y el 2,6\% al estrato cinco. En relación con el estado civil, $54,7 \%$ de las madres estaban en unión libre, $29,9 \%$ eran casadas y 15,4\% eran soleteras. En relación con el estado civil, 54,7\% de las madres estaban en unión libre, 29,9\% eran casadas y $15,4 \%$ eran soleteras. En cuanto a la escolaridad, se observó que el $34,2 \%$ de las madres tenían bachillerato completo, seguido de carreras técnicas con 32,5\%, universitarios $18,8 \%, 0,9 \%$ postgrado y un $13,8 \%$ restante para bachillerato incompleto, primaria completa e incompleta. De acuerdo con la ocupación, el $54,7 \%$ de las madres eran empleadas y un $45,3 \%$ amas de casa.

\section{Variables obstétricas}

De acuerdo con los criterios de inclusión, todas las madres tuvieron una gestación a término. El el $52,1 \%$, eran primigestantes, seguido de secundigestantes con un 33,4\% y multigestantes con $14,5 \%$. La principal vía del parto fue vaginal con $80,3 \%$, seguido de cesárea con $16,2 \%$ y parto atendido con fórceps para 3,4\%.

\section{Apego sano o apego en riesgo}

Se presentó un apego sano en el 80,3\% de las madres y un apego en riesgo en el 19,7\%. La conducta maternal donde se observó mayor frecuencia para el apego en riesgo fue la alimentación, sin embargo, a pesar de que no hubo asociación estadística, una alta proporción de las madres mostró una baja motivación hacia la lactancia materna, hicieron el intento de ofrecer el seno al bebé, pero requirieron de mucha ayuda y apoyo para hacerlo (Tabla 1 ).

Tabla 1. Variables conductuales: alimentación, lenguaje, tocar, interés y respuesta, según los puntajes de apego sano o en riesgo de las madres en postparto. Medellín 2014

\begin{tabular}{|c|c|c|c|c|c|}
\hline & \multicolumn{5}{|c|}{${\text { Variables conductuales según escala FIRST }{ }^{1}}^{1}$} \\
\hline & Alimentación & Lenguaje & Tocar & Interés & Respuesta \\
\hline Apego & n (\%) & $\mathrm{n}(\%)$ & $\mathrm{n}(\%)$ & $\mathrm{n}(\%)$ & $\mathrm{n}(\%)$ \\
\hline En riesgo & $71(60,7)$ & $41(35)$ & $25(21,4)$ & $30(25,6)$ & $26(22,2)$ \\
\hline Sano & $46(39,3)$ & $76(65)$ & $92(78,6)$ & $87(74,4)$ & $91(77,8)$ \\
\hline
\end{tabular}

\section{Análisis bivariado}

Al explorar la asociación entre el apego y las variables obstétricas y demográficas de interés se encontró asociación entre el estado civil (unión libre y soltera) y la escolaridad (bachillerato) con la variable dependiente. Las demás variables no presentaron asociación estadística (Tabla 2). 
Tabla 2. Asociación de variables demográficas con el apego en riesgo durante el postparto

\begin{tabular}{|c|c|c|c|}
\hline Variable & $\begin{array}{c}\text { Prevalencia apego en riesgo } \\
\text { n (\%) }\end{array}$ & RP, IC95\% & Valor $\mathrm{p}^{*}$ \\
\hline \multicolumn{4}{|l|}{ Estado civil } \\
\hline Soltero & $5(29,4)$ & $5,1(1,1-23,8)$ & 0,036 \\
\hline Unión libre & $16(24,6)$ & $4,3(1,1-17,7)$ & 0,043 \\
\hline Casado & $2(5,7)$ & & \\
\hline \multicolumn{4}{|l|}{ Estrato socioeconómico } \\
\hline Bajo & $17(27,9)$ & $2,1(0,9-4,72)$ & 0,061 \\
\hline Medio- alto & $6(10,7)$ & & \\
\hline \multicolumn{4}{|l|}{ Nivel de escolaridad } \\
\hline Primaria-Bachillerato & $16(28,1)$ & $2,85(0,75-10,78)$ & 0,121 \\
\hline Universitarios- otros & $14(24,5)$ & $2,35(1,03-5,38)$ & 0,043 \\
\hline \multicolumn{4}{|l|}{ Edad en años } \\
\hline$<19$ & $7(50,0)$ & $7(0,98-49,6)$ & 0.052 \\
\hline 20 a 34 & $15(16,8)$ & $2,35(0,33-16,4)$ & 0,387 \\
\hline 35 y más & $1(7,14)$ & & \\
\hline \multicolumn{4}{|l|}{ Ocupación } \\
\hline Ama de casa & $13(24,5)$ & $0,63(0,30-1,33)$ & 0,227 \\
\hline Empleada & $10(15,6)$ & & \\
\hline
\end{tabular}

\section{Análisis multivariado}

Tras ingresar las variables que cumplían con los criterio de Hosmer-Lemeshow, o que tenían plausibilidad biológica al modelo multivariado, se encontró que la única de ellas que explica la asociación con el apego en riesgo es el estado civil soltero, con una RP = 4.88 IC95\% $(1,01-23,45)$ y valor $\mathrm{p}=0,48$; las demás variables no mostraron asociación estadística con la variable dependiente.

\section{Discusión}

\section{Factores demográficos relacionados con el apego}

En el análisis bivariado del presente estudio se mostró que hubo asociación entre el apego en riesgo y el estado civil (unión libre y soltera). Mientras que el análisis multivariado, encontró que la única de ellas que explica la asociación con el apego en riesgo es el estado civil soltero, con una RP $=4.88$ IC95\% $(1.01 ; 23.45)$ y valor $p=$ 0.48 . Así mismo, el estudio de Salariya y Cater(2), sobre la relación temprana entre madres e hijo, en los primeros cinco días postparto, buscaron asociación con las variables demográficas y mostraron que ser madre soltera se asoció con el apego en riesgo en los días 1 y 5 postparto. (Primer día: $\mathrm{Chi}^{2}=7,3 \mathrm{p}=<0,01$, quinto día: $\mathrm{Chi}^{2}=$ $14,6 \mathrm{p}=<0,01$ ).

El estudio de Lecannelier et al.(12), evaluó los patrones de apego y observaron diferencias de porcentajes de las madres de estado civil en la categoría de unión libre en relación con las solteras y casadas, pero no hubo asociación entre estas variables con los patrones de apego. $\left(\mathrm{Chi}^{2}=\right.$ $0,500, p=0,779$ ). Refieren también que es probable que actúen otras variables más allá de la condición marital de la madre, tales como el grado de satisfacción en rol maternal, el compromiso maternal, el clima familiar, y el estado mental de la madre con respecto al apego.

En el ámbito de la relación de pareja, el estudio de Muñoz et al.(9), sobre la prevalencia del riesgo relacional entre la diada madre e hijo y variables asociadas, encontraron que la asociación con alto riesgo relacional resultó significativa para las 
siguientes variables: mujeres sin pareja estable, mujeres solteras o separadas y monoparentalidad. (Sin pareja estable: OR 5,56, $\mathrm{IC}=2,46-12,59$, Soltera: $\mathrm{OR}=3,75, \mathrm{IC}=2,29-6,14$, monoparentalidad $30 \mathrm{R}=, 26 \mathrm{IC}=1,83-5,08)$.

Un metanálisis realizado por BakermansKranenburg y van Ijzendoorn(13) sobre el apego seguro y familias de alto riesgo, concluyó que ser madre soltera puede limitar el acceso a los recursos financieros o sociales, aumentar el aislamiento social y el estrés materno y resultar en una conducta inadecuada de cuidado y en el apego desorganizado del niño. El estudio de Salazar et al.(14), realizado en un grupo de madres adolescentes solteras, refieren que el $33 \%$ de estas madres permanecieron solteras durante el embarazo y después de tener al hijo y que en estratos socioeconómicos bajos predominó el madre-solterismo.

Es importante reflexionar que la situación actual de madre-solterismo, cada vez es más frecuente y son diversas las causas por las cuales se ha dado origen a esta nueva tipología que marcan el desarrollo y dinámica de la familia en el aspecto afectivo, educativo y económico(17). A pesar de que hoy en día hay cambios importantes en la estructura familiar, se requiere darle una mirada a la necesidad de apoyo y ayuda a las madres solteras y con dificultades económicas, a través de una red social que contribuya en el proceso de cuidado de sus hijos desde los primeros momentos del nacimiento y crear estrategias de acción e intervención más acordes con las necesidades económicas, de afecto y amor en la crianza.

En cuanto al estrato socioeconómico y el apego, el presente estudio mostró que el estrato socioeconómico no presentó asociación con el apego en riesgo; este hallazgo es similar a los resultados de Muñoz et al.(9), quienes no encontraron asociación estadística con el nivel socioeconómico a pesar de que la mayoría de las madres pertenecen al estrato bajo $(77,7 \%)$, sin embargo, comentan que un alto porcentaje de mujeres que afrontan el embarazo y la crianza sin el apoyo económico aumenta la posibilidad de alto riesgo relacional.
El estudio de Lecannelier et al.(12), evaluó los patrones de apego y la asociación con algunas variables y encontró que el nivel socio-económico de las madres y el riesgo relacional no tuvo asociación estadística comparada con otros estudios donde el estrato social o las poblaciones más vulnerables desde el punto de vista social, tienen mayor frecuencia de apegos inseguros y desorganizados(13). En este sentido saca dos conclusiones que pudieron derivar estos resultados, que si bien la muestra evaluada provenía de estrato medio-bajo y bajo, los niveles de escolaridad encontrados no permitían afirmar que la muestra es de alta vulnerabilidad socioeconómica. La segunda es que puede deberse a los factores asociados a la calidad del apego como es el compromiso maternal(12).

Un estudio realizado sobre el apego seguro y familias de alto riesgo(13), destacó que la inseguridad de apego de los niños y la desorganización de los apegos, se ven fuertemente afectados por el maltrato de los padres y por la condición socioeconómica. Estas condiciones sociales suponen un riesgo para las interacciones tempranas entre las madres y sus recién nacidos, con consecuencias adversas en el bienestar individual del infante a largo plazo y el del contexto familiar con efectos en la salud poblacional, así mismo como lo expresa Palacio Hernández(10).

La investigación de Ortiz et al.(15), refiere que la población de estrato socioeconómico bajo, tiene mayor frecuencia de violencia intrafamiliar y un menor número de niños con apego sano. Así mismo, la investigación de Lickenbrock y Braungart-Rieker(5), sobre el apego sano, concluye que no solamente la sensibilidad de las madres se relaciona con el apego, sino también otros factores como el nivel socioeconómico, que afecta a la calidad de la crianza y el comportamiento social de los niños. Dubber et $a l .(6)$, indican que el efecto de la sensibilidad de las madres, también dependió de los niveles de recursos económicos de la familia y que frente a condiciones más difíciles como: los padres más jóvenes, menos educados y con ocupaciones menos remunerados, existe mayor tendencia al apego en riesgo. Los factores como la falta de 
autonomía económica y la baja educación perpetúan la pobreza, lo cual puede establecer patrones de crianza con riesgo de estabilidad emocional(8).

En cuanto a la variable de escolaridad y su asociación con el apego encontrada en el presente estudio, se encontró asociación para la escolaridad bachillerato RP, IC95\% 2,35 (1,03 $5,38)$ p $>0,005$. El estudio de Koroleff y Nóblega7 mostró que las madres sin ninguna instrucción o educación relacionada con la interacción con su recién nacido, tenían bajas puntuaciones para un adecuado apego, donde el $46,2 \%$ de ellas tenía secundaria completa. Así mismo lo afirmó el estudio de Escobar ${ }^{5}$ y el estudio de Dubber et al.(6), quienes refieren que las madres menos educadas tienen una mayor tendencia al apego en riesgo, pero no hubo significancia estadística.

El estudio sobre la evaluación de patrones de apego de Lecannelier et al.(12), refiere que en cuanto a la escolaridad de las madres, el mayor porcentaje corresponde a educación media completa $(39,7 \%)$, seguido de un $20,6 \%$ de madres con escolaridad media incompleta, el 19,9\% educación técnica o universitaria, y el 9,2\% con educación básica incompleta. Al cruzar la variable educación y apego, refieren que no hubo significancia estadística.

Otra de las variables demográficas estudiadas fue la edad, la cual en el presente estudio no tuvo asociación estadística ( $<19$ años RP, IC 95\% $7(0,98-49,6)$; p 0.052 y 20 a 34 años RP, IC $95 \%$ $2,35(0,33$ - 16,4); p 0,387). Sin embargo, las madres de menor edad presentaron proporciones más altas para el apego en riesgo $(<19$ años con $50 \%$ y 20 a 34 años con 16,8\%). De la misma manera lo muestra el estudio de Lecannelier et al.(12), quienes analizaron la distribución de patrones de apego en una muestra de 130 madres con sus hijos y las posibles relaciones con las características sociodemográficas, tales como la edad, escolaridad y estado civil. Encontraron que no hubo asociación entre la edad de las madres y los patrones de apego, no obstante, las madres adolescentes presentaron el porcentaje más alto de apego inseguro que el resto, aun cuando esta diferencia no fue estadísticamente significativa
$\left(\mathrm{Chi}^{2}=2,736 \mathrm{p}=0,255\right)$ y el grupo de madres de 37 a 46 años, presentaron una mayor tendencia hacia el apego seguro (87\%).

El estudio de Muñoz et al.(9), encontró que ser madre adolescente entre 14 y 19 años, está asociado con un alto riesgo relacional con su hijo $(\mathrm{OR}=1,89 \mathrm{IC}=1,10-3,23)$. Dichos autores señalan que generalmente estas madres, presentan una actitud de rechazo y ocultamiento del embarazo por temor a la respuesta del grupo familiar, lo que conllevaría un control prenatal tardío o insuficiente que dificultaría la detección de problemas o la implementación de medidas de intervención precoz. También mencionan que en el área cognitiva, las adolescentes muestran un pensamiento egocéntrico, centrado en sí mismas, orientadas a lo inmediato y a la satisfacción de sus necesidades, que las incapacita para generar sintonía con su hijo.

Otros estudios(15,16), plantean que las relaciones afectivas madre-hijo se dificultan en las madres más jóvenes, pues se enfrentan a las tareas que demanda la maternidad a una edad en la que no se encuentran preparadas para desempeñarlas. Agregan que la adopción abrupta del rol de madre, genera en muchas jóvenes diversos conflictos que interfieren con sus habilidades maternales y les dificultan la posibilidad de proveer un ambiente socioemocional adecuado para el niño. Las madres más jóvenes, tienen dificultades para regular sus estados emocionales, lo cual se evidencia en altas tasas de depresión y de cambios afectivos. Estos estados hacen que sean menos sensitivas con sus hijos, estén menos disponibles emocionalmente y promuevan el establecimiento de vínculos afectivos inadecuados con sus hijos.

\section{Frecuencia de apego sano y en riesgo}

Es importante considerar que al revisar la frecuencia de apego en los países de Latinoamérica, se ha identificado una muy baja producción científica en artículos relacionados con la prevalencia del apego materno infantil, así como lo comenta el estudio de Palacio Hernández ${ }^{10}$, quien hizo una revisión entre 1962 hasta 2016 y encontró que sólo se tiene el 3\% de la producción mundial. En este sentido con tan 
poca información, se dificulta el conocimiento sobre las alteraciones en el proceso de apego, así como de los factores de riesgo relevantes en esta región. Sin embargo, el estudio en mención encontró una prevalencia de trastornos del apego materno-infantil desde el 22 al $67 \%(10)$.

Para hallar la frecuencia del apego sano o en riesgo en el presente estudio, se evaluó las conductas maternas a través de la escala de medición del instrumento Mother-child relationship - FIRST score(2). Los resultados mostraron que las madres hospitalizadas en postparto, en general tuvieron un apego sano con su recién nacido; datos que coinciden con el estudio de Escobar(8) quien analizó los patrones de apego en madres adolescentes y evaluó el riesgo relacional o sea el conjunto de conductas y actitudes maternales que indican un proceso de apego en riesgo y encontró que el $60 \%$ de las madres adolescentes presentó bajo riesgo relacional y el $40 \%$ restante, presentaron alto riesgo relacional. De manera similar el estudio de Lecannelier et al.(12), sobre los patrones de apego en infantes, en términos de apego seguro e inseguro, obtuvo como resultado que la proporción de apego seguro es de 73,08\%, y la de apego inseguro es de $26,92 \%$.

El estudio de Muñoz et al.(9), sobre el riesgo relacional entre madre y recién nacido, encontró que la prevalencia de alto riesgo relacional fue del $43,8 \%$. El riesgo relacional por estos autores es definido por la interacción disarmónica, fría, no contingente, insensible y desintonizada que se puede expresar en ausencia de contacto físico, verbalizaciones y contacto visual; falta de competencia en la muda, la alimentación y al calmarlo; intolerancia frente al llanto y falta de aceptación de las características básicas del bebé. Este concepto es muy similar al que empleó el presente estudio para definir el apego en riesgo y así mismo lo expresan Kimelman et al.(17), en su investigación sobre riesgo relacional entre madre y recién nacido.

Así mismo, evaluó cinco conductas indicadoras de apego desde la escala Mother-child relationship - FIRST score(2), con el fin de definir cuáles madres se encontraban en apego sano o en riesgo, se mostró que la mayoría de las madres $(60,7 \%)$, tuvieron bajas puntuaciones para la lactancia, es decir, se observó poca motivación e hicieron el intento de ofrecer el seno al bebé, pero requirieron de mucha ayuda y apoyo para hacerlo, sin embargo, no hubo significancia estadística. Es importante destacar este resultado por considerarse la lactancia como el momento primordial para el inicio del apego y donde se establece una comunicación intensa entre madre e hijo(18-20). En este aspecto, los profesionales de la salud desempeñan un rol crítico para brindar apoyo y acompañamiento, a la vez que pueden influenciar las decisiones relacionadas con las prácticas de lactancia de las madres y las familias(20).

\section{Conclusiones}

Hubo asociación entre el estado civil (unión libre y soltera) y la escolaridad (bachillerato) con el apego en riesgo, sin embargo el apego en riesgo se explica en el modelo multivariado solamente con el estado civil soltero.

A pesar de que lactancia materna fue considerada como una fuente fundamental de apego, hubo una alta proporción de madres con baja motivación hacia ella y además, requirieron de mucha ayuda para iniciarla.

\section{Recomendaciones}

Capacitar al personal de salud en todas las variables que incentivan el apego sano y aquellas que lo afecten, para que puedan identificarlas en el postparto.

Establecer un protocolo de cuidado para la lactancia materna que incluya la técnica, otras formas de ofrecer la leche materna cuando es difícil pegar al bebé del seno y enseñar a la madre, la probabilidad de los riesgos para la salud del niño, si esta no se lleva a cabo.

Durante el control prenatal, identificar aquellas madres solteras y con dificultades económicas para que sean referidas a los entes pertinentes, con el fin de beneficiarse de las políticas, estrategias e intervenciones de salud pública $u$ otros programas alusivos. 
En este sentido, para el desarrollo del apego, es necesario que los profesionales de la salud en los servicios de postparto, reconozcan éstas características, en donde el papel de enfermería es central para determinar la capacidad de respuesta sensible de la madre con su hijo y fortalecer todas las variables que incentivan el apego sano y reconocer aquellas que lo afectan.

\section{Conflicto de intereses}

Ninguno declarado por los autores.

\section{Referencias}

1. Marinus VI. El Apego durante los Primeros Años (0-5) y su Impacto en el Desarrollo Infantil. Países Bajos Leiden Univ [Internet]. 2010;1-5. Disponible en: http://www.enciclopediainfantes.com/sites/default/files/textesexperts/es/2283/el-apego-durante-los-primeros-anos0-5-y-su-impacto-en-el-desarrollo-infantil.pdf

2. Salariya EM CJ. Mother-child relationship--first score. J Adv Nurs. 1984;9(6):589-95.

3. Daniela CM. Descripción de los patrones de apego de madres con depresión postparto y sus hijos lactantes de entre 12 y 15 meses. 2008.

4. Bowlby J. El vínculo afectivo. Paidos. 1976;

5. Lickenbrock DM B-RJ. Examining antecedents of infant attachment security with mothers and fathers: an ecological systems perspective. Infant Behav Dev. 2015;39:173-187.

6. Dubber S, Reck C, Müller M, Gawlik S. Postpartum bonding: the role of perinatal depression, anxiety and maternal-fetal bonding during pregnancy. Arch Womens Ment Health. 2015;18(2):187-95.

7. Aguilar C, Knapp M, Moscoso MS, Pollitt E. Promoviendo vínculos saludables entre madres adolescentes y sus bebes una experiencia de intervención. Rev Psicol. 2010;28(2):259-82.

8. Escobar M. Historia de los patrones de apego en madres adolescentes y su relación con el riesgo en la calidad del apego con sus hijos recién nacidos [Tesis maestría]. Santiago: Universidad de Chile, Facultad de Ciencias Sociales; 2008.

9. Muñoz M, Poo AM, Baeza B, Luis BM. Riesgo relacional madre-recién nacido: estudio de prevalencia y variables asociadas. Rev Chil Pediatr. 2015;86(1):25-31.

10. B. P-HB. Alteraciones en el vínculo materno- infantil: prevalencia, factores de riesgo, criterios diagnósticos y estrategias de evaluación. Rev Salud UIS. 2016; Vol. 48:164-76.

11. Ministerio de Salud. Resolución 8430 de 1993. Ministerio de Salud y Protección Social, República de Colombia. 1993 p. 1-19.

12. Lecannelier F, Kimelman M, González L, Nuñez C, Hoffmann M. Evaluacion de patrones de apego en infantes durante su segundo año en dos centros de atencion de Santiago de Chile. Rev Argentina Clin Psicol. 2008;17(3):197-207.

13. Bakermans-Kranenburg MJ, van IJzendoorn $M H$, Kroonenberg PM. Differences in attachment security between African-American and white children: Ethnicity or socio-economic status? Infant Behav Dev. 2004;27(3):417-33.

14. Salazar Arango A, Acosta Murcia MM, Lozano Restrepo N, Quintero Camacho MC. Consecuencias del embarazo adolescente en el estado civil de la madre jóven: Estudio Piloto en Bogota, Colombia. Pers Bioética [Internet]. 2008;12:169-82. Disponible en: http://www.scielo.org.co/pdf/pebi/v12n2/v12n2a08. pdf

15. Ortiz JA, Borré A, Carrillo S, Gutiérrez G. Relación de apego en madres adolescentes y sus bebés canguro. Rev Latinoam Psicol. 2006;38(1):71-86.

16. Gutiérrez M, Castellanos S, Henao J, Santacoloma A. La atmósfera psíquica y los vínculos significativos de madres adolescentes gestantes y lactantes de bajo estrato socio-económico. Implicaciones sobre el desarrollo psíquico. Rev. latinoam. cienc. soc. niñez. 2007;5(1):1-24.

17. Kimelman M, Núñez C, Hernández G, Castillo N, Páez J BS. Construcción y evaluación de pauta de medición de riesgo relacional madre-recién nacido. Rev Méd Chile. 1995;(123):12-707.

18. Noceda LAC. El apego. Su importancia para el pediatra. Pediatría (Asunción) [Internet]. 2012;39(3):199-206. Disponible

en: http://www.revista.spp.org.py/index.php/ped/article/ view/16/35

19. Hernández G, Kimelman J MO. Salud mental perinatal en la asistencia hospitalaria del parto y puerperio. Rev Méd Chile. 2010;128(11):1283-9.

20. OPS O. La alimentación del lactante y del niño pequeño Capítulo Modelo para libros de texto dirigidos a estudiantes de medicina y otras ciencias de la salud. 2010. p. 85-8. 